\title{
UM ESTUDO DE COMO O PROCESSO DE COACHING PODE SER UM ALIADO NO TRATAMENTO DO CÂNCER
}

\section{A STUDY OF HOW THE COACHING PROCESS CAN BE AN ALLY IN CANCER TREATMENT}

Cristiane S. dos Santos Lima

Aluna do MBA em Gestão Estratégica de Pessoas da Universidade Fumec. BH/MG/Brasil

\section{Silvana Chiaretto}

Professora e orientadora do MBA em Gestão Estratégica de Pessoas da Universidade Fumec. BH/MG/Brasil. Email: silvana.chiaretto@fumec.br. 


\title{
RESUMO
}

O processo de coaching como um aliado no tratamento oncológico é o tema de estudo deste artigo, tendo como objetivo principal compreender como o coaching influência e impacta na vida das pessoas em tratamento do câncer. Como metodologia de estudo, foi efetuada uma revisão bibliográfica sobre o tema e uma pesquisa telematizada. Observou-se que a busca por uma autorrealização, apoio efetivo e motivação é uma necessidade que acompanha pessoas em tratamentos oncológicos os quais os profissionais da saúde estão longe de suprir. Ao final do estudo considerou-se que o coaching pessoal pode trabalhar na área de saúde para ajudar o paciente a progredir no tratamento. Para tanto, considera-se que o processo de coaching é um suporte para superar grandes dificuldades na busca por um objetivo ou meta. Sendo assim, o coaching pode ser uma ferramenta de impacto positivo, de apoio e mudança de hábito, na vida de pessoas em tratamento do câncer.

Palavras-Chave: Coaching, motivação, tratamento de câncer.

\begin{abstract}
The coaching process as an ally in cancer treatment is the topic of study in this article, with the main objective to understand how coaching influences and impacts the lives of people undergoing cancer treatment. As a study methodology, a bibliographic review on the subject and a telematized research were carried out. It was observed that the search for self-realization, effective support and motivation is a need that accompanies people in cancer treatments that health professionals are far from meeting. At the end of the study, it was considered that personal coaching can work in the health field to help the patient progress in treatment. For that, it is considered that the coaching process is a support to overcome great difficulties in the search for an objective or goal. Therefore, coaching can be a tool with a positive impact, support and change of habit, in the lives of people undergoing cancer treatment.
\end{abstract}

Keywords: Coaching, motivation, cancer treatment. 


\section{INTRODUÇÃO}

Recentemente, o processo de coaching está ganhando cada vez mais espaço na área de Gestão de Pessoas nas universidades e nas organizações. Além de tema muito comentado e discutido no Brasil e no mundo nos últimos anos, o coaching pode ser considerado hoje, como a metodologia mais buscada para empresas que almejam alcançar resultados positivos em diversas áreas em curto prazo $(\mathrm{G} 1,2018)$.

Este estudo se justifica por perceber a demanda pelo processo de coaching também na área da saúde. Percebe-se a aplicação do processo de coaching na educação, orientação e treinamento para estimular a saúde e elevar o bem-estar das pessoas e visa incentivar a independência do paciente/coachee fazendo-o conhecedor do seu próprio organismo e do seu cérebro sobre a excelente realização das funções vitais segundo Marques (2015).

Entretanto é importante salientar que, o tema escolhido desperta nas autoras amplo interesse em defender a aplicabilidade do processo de coaching no âmbito da saúde, haja vista que, o processo de coaching possui características importantes transformação do indivíduo segundo Gaspar \& Portásio (2009).

Sendo assim, percebe-se a necessidade de um melhor acompanhamento às pessoas diagnosticadas com câncer e que muitas vezes se sentem desorientadas e desmotivadas mediante ao primeiro diagnóstico. O que impulsionou a realização deste trabalho foi compreender que o procedimento de um tratamento oncológico exige muito mais do que mudanças na rotina alimentar e física, exige apoio por parte dos profissionais, força, determinação, coragem e impulso por parte dos pacientes e que pode mudar todo este período inicialmente conturbado para uma fase mais aceitável e motivada.

Para tanto, é preciso problematizar o coaching e tentar compreender como o processo pode influenciar e impactar positivamente no tratamento do paciente com câncer.

Para tanto, este estudo possui várias hipóteses: $\left(1^{\mathrm{a}}\right)$ o processo de coaching pode melhorar a motivação de pacientes diagnosticados com câncer? $\left(2^{\mathrm{a}}\right) \mathrm{O}$ coaching pode utilizar ferramentas necessárias e de elevado suporte para orientar e acompanhar os pacientes antes, durante e após o tratamento? ( $\left.3^{\mathrm{a}}\right)$ Ele pode estimular a qualidade de vida dos pacientes, levando em conta a mudança de hábitos rotineiros com a implementação de novas habilidades e competências? $\left(4^{\mathrm{a}}\right) \mathrm{O}$ coaching pode ajudar nas questões pessoais tais como: vida familiar, profissional e manter sempre em equilíbrio a motivação em busca dos resultados esperados? 
$\left(5^{a}\right)$ Um questionário aplicado aos pacientes pode ser uma forma de orientação a respeito de como o coaching vai buscar as informações necessárias e utilizar as ferramentas certas como aliado ao tratamento de câncer?

Sendo assim, o estudo tem como objetivo geral; compreender como o coaching pode influenciar e impactar no tratamento ao câncer. E como objetivos específicos: (1) decompor os conceitos de coaching; (2) analisar se a metodologia contribuiu no estímulo da motivação dos pacientes oncológicos; (3) averiguar se o processo de coaching pode auxiliar no tratamento de pacientes com câncer; (4) verificar quais são as práticas e ferramentas que mais contribuem no estímulo da motivação de pacientes com câncer; (5) avaliar os benefícios do processo de coaching na melhora do paciente com câncer.

Dessa forma, as metodologias de pesquisa foram: $\left(1^{\circ}\right)$ levantamento bibliográfico e $\left(2^{\circ}\right)$ pesquisa tematizada. Ambas acompanhadas dos tipos de pesquisa: qualitativa e descritiva. Como técnicas de coleta de dados foram utilizadas as análises bibliográfica e telematizada. E como técnica de tratamento dos dados foi utilizada a análise de conteúdo.

Em relação à estrutura do artigo: o capítulo um se refere à introdução, o capítulo dois apresenta o referencial teórico e foi dividido em 5 tópicos: conceitos de coaching, evolução do coaching, ferramentas de coaching, as influências do coaching na vida pessoal, evolução do câncer no Brasil, o coaching e o tratamento do câncer no Brasil. O capítulo três se refere à metodologia de pesquisa seguida do capítulo quatro, onde constam a análise e resultados. No capítulo cinco se apresentam as considerações finais e, por último, as referências.

Por fim, este estudo buscou-se a responder o seguinte questionamento: como o processo de coaching pode ser um aliado no tratamento do câncer?

\section{REFERENCIAL TEÓRICO}

Neste capítulo serão exibidos os conceitos de coaching, a evolução do coaching, ferramentas de coaching, as influências do coaching na vida pessoal, o coaching e o tratamento do câncer no Brasil. 


\subsection{Coaching: conceitos}

Segundo Lotz \& Gramms (2014), o coaching é uma metodologia que incentiva o indivíduo a pensar mais e desenvolver seu potencial e aprendizado para o sucesso no seu desenvolvimento profissional e pessoal.

Pelas perspectivas de Boog \& Boog (2013), o principal ponto do coaching é a assistência, a preparação e o suporte do coach (aquele cujo nome é dado como se fosse uma espécie de treinador) para a realização do que foi estipulado para seu cliente (aqui ele é conhecido como coachee, ou seja, a pessoa que é treinada), seja na área profissional, pessoal ou até mesmo social para serem trabalhados em curto ou médio prazo.

O coaching também pode ser entendido como "uma prática de relacionamento que envolve dois profissionais: o coach e o coachee" (MUNHOZ, 2017, p.31).

Lubk (2016), afirma que o coaching surge a partir de uma série de questionamentos e reflexões onde o profissional (coach) aconselhará o seu cliente (coachee) a expor o caminho mais propenso a seguir para alcance dos objetivos.

Pelos conceitos dos autores aqui mencionados, pode-se inferir que o coaching é uma atividade cujo objetivo principal é dar assistência, aconselhamentos e orientações necessárias para que os clientes, ou coachees, possam obter sucesso em suas vidas profissionais, pessoais e sociais.

\subsubsection{Evolução do Coaching}

Em meados do século XVII Costa \& Caregnatto (2012) descrevem que as corridas realizadas pelas carruagens eram conhecidas por coaching, conduzidas pelos coachman e representavam um ícone da elite. Contudo, Ferreira (2008) salienta que esse termo foi se expandindo a partir da França alcançado outras fronteiras como Inglaterra, e se popularizando na Europa e América do Norte.

Ao longo dessa trajetória Costa \& Caregnatto (2012) descrevem que a pronúncia do termo coaching sofreu algumas alterações de acordo com as línguas, mas o seu significado se manteve, logo o primeiro uso no idioma inglês era referente a carruagem em questão. Diante disso, o coaching tem o significado de conduzir uma pessoa preciosa de um determinado lugar até aonde ela deseja estar. 
Outra corrente atrelada a origem do coaching de acordo com Gaspar \& Portásio (2009) está relacionada aos primeiros estudos sobre liderança, no qual iniciaram antes da época da burguesia, uma vez que, nesse período já tinham grupos direcionados a resolução de determinadas tarefas, onde eram praticadas em sistemas não capitalistas e produziam grandes resultados.

Em 1950, o coaching foi usado como uma técnica de gerenciamento de pessoas e utilizado nos primeiros procedimentos de desenvolvimento pessoal (MARQUES, 2013).

No entanto, outra corrente descreve que o termo coaching originou-se a partir do âmbito esportivo, e é uma das principais características atrelada a sua origem, nesse âmbito Josefa (2015) comenta que em 1860, surgiu um técnico desportivo denominado de coach.

Já no século seguinte em 1980 nos Estados Unidos o professor de tênis Timothy Gallwey deu origem a história que chega até os dias atuais, através do livro The Inner Game of Tenis, no qual ele desenvolveu uma metodologia a fim de que as pessoas aprimorassem suas práticas desportivas para atingirem melhores resultados. Os métodos utilizados por Gallwey introduziram a idealização de que um coach eficiente poderia ajudar o jogador a reduzir ou remover os obstáculos internos ligados a atuação de sua performance, além de auxiliá-lo na concentração e autoconfiança bem como acreditar que o ser humano é sempre capaz de ser melhor a cada dia.

Atualmente, "o coaching muito utilizado no ambiente organizacional, acadêmico e profissional, pode ser aplicado como um programa interno na formação de líderes, ou mesmo quanto ao alcance dos resultados no cumprimento de metas pessoais" (BOOG \& BOOG, 2013, p.401).

\subsubsection{Ferramentas de Coaching}

Boog \& Boog (2013) mencionam que cada escola possui suas próprias ferramentas e por isso não há uma conformidade quanto as ferramentas ou instrumentos de coaching.

Ainda segundo os autores Boog \& Boog (2013), no Brasil existem várias escolas com suas ferramentas próprias para atuação: coaching sistêmico, coaching ontológico, coaching com PNL, coaching terapêutico, coaching comportamental, holos coaching e mentoring, entre outros.

As principais ferramentas de coaching segundo Da Matta (2013) são: (a) Psicologia Positiva: essa ferramenta auxilia o coach a definir metas concretas e que é possível seu cliente 
chegar aos objetivos sendo desenvolvidos de maneira positiva e prazerosa. (b) Patrocínio Positivo: através dessa ferramenta o coach incentiva e mantém um laço de confiança com seu cliente; (c) Swot Pessoal: o coach utiliza essa ferramenta para mostrar ao coachee seus pontos fortes e fracos, suas habilidades, forças e fraquezas e a partir daí trabalhar e desenvolver seus principais problemas. (d) Missão e Visão: essa ferramenta contribui para que o coach não somente conheça seu cliente traçando suas metas, valores, os problemas e onde ele almeja chegar, como também a partir daí começa a desenvolvê-lo no alcance dos resultados. (e) Rota de Ação: após conhecer os clientes, essa ferramenta será auxiliadora para o coach traçar metas de curto e longo prazo para alcance de resultados.

Já Marques (2016) cita outras ferramentas do processo de coaching que podem complementar o rol de ferramentas de Da Matta (2013). São elas: (1) Avaliação de Perfil Comportamental: representados pelos animais gato, tubarão, lobo ou águia, cujos perfis comportamentais são marcantes, ajuda a identificar o perfil de cada pessoa. (2) Roda da Vida: essa ferramenta permite o conhecimento da vida de uma pessoa em todas as áreas. (3) Autofeedback: o coach utiliza essa ferramenta para traças os pontos fortes e fracos e outros fatores que levarão o coachee a autorreflexão para alcance dos objetivos.

De acordo com Boog \& Boog (2013), cada profissional de coaching utiliza um rol de ferramentas. O rol de ferramentas é determinado pela escola de formação do coach. É importante salientar que, muitas vezes o profissional de coaching, por meio de experiências e históricos de seus clientes, acabará por desenvolver seu próprio rol de ferramentas.

\subsubsection{Influências do coaching na vida pessoal}

Marques (2016) afirma que para pessoas que estão em busca de mudanças comportamentais duradouras em suas vidas pessoais, o coaching é a melhor metodologia e considerada mais efetiva, pois trabalha em todas as dimensões do ser humano de maneira que cada um possa progredir e melhorar seu potencial.

Ainda, segundo Marques (2016), o coaching empenha-se em auxiliar a pessoa a discernir os seus comportamentos mais restritivos, buscando onde teve início tais atitudes e como prejudicam sua vida.

A cada etapa, o cliente, ou mais precisamente, o coachee, poderá dedicar-se a cuidar de cada modo de comportamento, e próximo ao seu coach, elaborar e programar estratégias para acabar com as causas do que vem sendo possivelmente, um fracasso na vida pessoal. Os resultados são calculados a cada encontro e o coachee vai 
percebendo como a mudança de atitude traz resultados rápidos e distintos para sua vida (MARQUES, 2016).

Para D’Addario (2016) o coaching pessoal ou coaching de vida, lida com metas pessoais presentes em cada indivíduo. E através dessas metas é que são trabalhados o potencial de cada um levando em consideração que cada indivíduo é diferente um do outro e possui personalidades distintas.

O coaching pessoal é etapas de mudanças que coloca o indivíduo estando ele em uma situação atual insatisfatória, a um patamar que deseja conquistar (ONEDA, 2016).

Para França (2017) o coaching pessoal gera um processo de mudança, pois cria no coachee o hábito da reflexão. E para que isso aconteça, o indivíduo deve rever sua forma de pensar observando sua conduta no dia a dia e, então, o próprio indivíduo começa a se esforçar para alcançar as melhorias.

\subsection{Evolução do câncer no Brasil}

Segundo o BBC o câncer é uma das principais causas de morte em todo o mundo. Atualmente "cerca de 32 milhões de pessoas convivem com a doença e a estimativa é que o número de novos casos continuará aumentando apesar dos investimentos na batalha contra a doença" (BBC, 2016, s/p).

De acordo com o Jornal do Brasil [JB] (2018, s/p) “a doença poderá aumentar em 78,5\% até o ano de 2040 com um total de 998 mil novos casos registrados”.

Segundo Bassette (2018, s/p) "Em 2015, o Brasil registrou 209.780 mortes por câncer e 349.642 em relação a doenças cardiovasculares e do aparelho circulatório. Ao comparar esses números com os do ano de 1998, vê-se um aumento aceitável na mortalidade por neoplasias: 90\% de aumento relativo a 1998, onde 110.799 pessoas morreram da doença”.

Segundo Malzyner \& Caponero (2013) o controle do câncer no Brasil começou no início do século XX, mais precisamente na cidade do Rio de Janeiro, onde em 1920 foi criado o Departamento Nacional de Saúde Pública o qual regulou os serviços de saúde no território nacional.

Em 1924, durante o II Congresso Brasileiro de Higiene, observou-se que enquanto os índices de tuberculose baixavam o de câncer tendia a aumentar a cada década. Daí surgiu a necessidade de se tomar medidas preventivas. 
Em 1938, cria-se o INCA (Instituto Nacional do Câncer) e é inaugurado o centro de cancerologia no Serviço de Assistência Hospitalar do Distrito Federal, no Rio de Janeiro.

Durante todo o século XX foram inaugurados instituições e centros de pesquisa ao câncer no Brasil e atualmente, são milhares de grupos de ajuda, associações e hospitais especializados espalhados em todo o território nacional de acordo com Malzyner \& Caponero (2013).

\subsection{O coaching e o tratamento de câncer no Brasil}

Não é novidade que a saúde pública no Brasil passa por um período conturbado de má gestão. Pessoas além de serem mal atendidas, sofrem discriminação dos profissionais de saúde com a falta de médicos e a longa espera por exames e consultas (SOBRINHO, 2018).

O tratamento faz parte do programa nacional de controle do câncer e cujo objetivo principal é a cura, o prolongamento de vida útil e a melhoria da qualidade de vida. $\mathrm{O}$ tratamento só é dado após resultados de exames, o qual definirá o tipo de tratamento adequado a cada paciente (SPEECHLEY \& ROSENFIELD, 2000).

O processo de coaching pode ser benéfico não somente quando a doença já estiver instalada. Pelo contrário, o processo de coaching deve ser muito bem-vindo na prevenção da doença. "A aplicação do Coaching inicia durante o tratamento ou mesmo na prevenção das doenças. É nesse ponto que a atuação de um coach é eficaz” (MARQUES, 2015, s/p).

O coaching é essencial para que o ser humano se desenvolva de maneira saudável, mediante princípios morais, tolerável e de maneira a conceder autonomia (MATOS, 2017).

Pode-se inferir que o coaching não somente é uma metodologia utilizada em organizações, como também, quando utilizado na área da saúde, promove um alcance de resultados satisfatórios e uma melhor qualidade de vida aos pacientes.

\section{METODOLOGIA}

De acordo com o QUADRO 1 o método adotado pelos pesquisadores foi o levantamento bibliográfico que segundo Prodanov \& Freitas (2013) refere-se a este tipo de pesquisa quando elaborada a partir de material já publicado podendo ser livros, revistas, publicações em periódicos e artigos científicos, jornais, boletins, monografias, dissertações, teses, material cartográfico, internet, verificando a veracidade dos dados obtidos. 
Foram levantados conteúdos já publicados sobre o coaching como aliado ao tratamento de doenças e os demais assuntos relacionados ao tema.

QUADRO 1 - Métodos e técnicas utilizados na pesquisa

\begin{tabular}{|c|c|c|c|}
\hline Método & Tipo de Pesquisa & $\begin{array}{l}\text { Técnica de Coleta } \\
\text { de Dados }\end{array}$ & $\begin{array}{l}\text { Técnica de } \\
\text { Tratamento de Dados }\end{array}$ \\
\hline $\begin{array}{l}\text { Levantamento } \\
\text { bibliográfico } \\
\text { telematizado }\end{array}$ & Qualitativa descritiva & $\begin{array}{l}\text { Análise bibliográfica } \\
\text { Análise telematizada }\end{array}$ & Análise de conteúdo \\
\hline
\end{tabular}

Fonte: elaborado pelos autores (2018).

Concernente a pesquisa qualitativa descritiva, esta não propõe o uso de métodos e técnicas estatísticas e os pesquisadores analisam os dados por indução. O ambiente e o objeto de estudo em questão, são contato direto do pesquisador, necessitando de um trabalho mais rigoroso de campo (PRODANOV \& FREITAS, 2013).

Ainda segundo Prodanov e Freitas (2013) os dados coletados nessas pesquisas são descritivos, onde realizam a análise, o estudo, o registro e a interpretação dos fatos do mundo real e físico sem a interferência do pesquisador e que geralmente os dados são usados em pesquisas de opinião.

À coleta de dados, utilizou-se a análise bibliográfica que como vantagem principal oferece maior série de fenômenos ao pesquisador ao invés daqueles que poderia pesquisar diretamente (GIL, 2008).

Contudo, há uma bibliografia adequada disponível ao pesquisador, livre de grandes dificuldades para contar com as informações requisitadas. Isso se torna importante quando o problema de pesquisa tem a ver com dados muito disseminados no espaço. Na maioria dos casos, não se compreende outra forma de conhecer os fatos se não com base em análises de bibliográficas (GIL, 2008).

O tratamento de dados foi feito por meio de análise de conteúdo entendida como um conjunto de técnicas de pesquisa com o objetivo, a busca do sentido ou dos sentidos de um documento (CAMPOS, 2004). 


\section{ANÁLISE E RESULTADOS}

O estudo abordado investigou de forma minuciosa e buscou apresentar um estudo de revisão bibliográfica e demonstrar como o coaching pode ser uma importante ferramenta aliada ao tratamento de câncer. O método de categorização foi utilizado com a finalidade de detalhar cada proposta pesquisada.

\subsection{Categoria de análise 1: o uso da metodologia coaching na vida pessoal}

Segundo Silva (2012) o Coaching é um vínculo de suporte prestado por um coach que disponibiliza os meios essenciais para que os clientes/coachees estimulem os recursos de que dispõem ajudando-os, assim, encontrar as respostas que procuram para os problemas relatados.

Para Marques (2016) o Coaching é visto muito mais que um processo ou método. Ele é visto como um incentivo e um suporte para aqueles que buscam mudanças favoráveis e duráveis em qualquer campo da vida. E uma pessoa pode passar por várias situações em sua vida em que é comum ficar desorientado, ficar perdido e não conseguir estabelecer metas e objetivos de sucesso.

Ao analisar os conteúdos bibliográficos, percebeu-se que coaching se adequa a qualquer situação pessoal e para essas ocasiões o Coaching Pessoal pode ser a resposta para pessoas que desejam mudar hábitos, ajudar a equilibrar o emocional, e melhorar a saúde, por exemplo.

Verificou-se igualmente, que o coaching pessoal pode trabalhar na área de saúde e mostra como o coach ajuda seu coachee a progredir em alguma etapa da vida. O coaching é um suporte para superar grandes dificuldades na busca por um objetivo ou meta.

\subsection{Categoria de análise II: o paciente oncológico e o conhecimento da doença e dos direitos legais}

Um estudo realizado pelos autores Sonobe, Buetto \& Zago (2011) com 42 pacientes no Núcleo de Oncologia da Sociedade Beneficente e Hospitalar Santa Casa de Misericórdia de Ribeirão Preto - SP, mostrou que 93\% desses pacientes eram conhecedores do diagnóstico sobre a doença, mas nenhum deles demonstraram conhecimento sobre todos os direitos do paciente com câncer, ou seja, apenas alguns conhecimentos. E que a forma como $48 \%$ dos pacientes adquiriram informações sobre seus direitos foram por outros meios, não pelos profissionais da saúde. 
Nota-se que mesmo após os pacientes saberem de seus direitos legais, muitos não se manifestaram na busca dos mesmos. A desmotivação em que muitos se sentem frente aos processos demorados e a forma que muitos passam como despercebidos pelos órgãos públicos, os fazem desistirem antes mesmo da conquista de algum benefício.

\subsection{Categoria de análise III: o Coaching para auxiliar pessoas diagnosticadas com câncer}

Chamado de Onco Coaching, cujo objetivo é auxiliar no tratamento do câncer, esse processo contribui causando maior segurança e idealizando planos e metas reais de cura na vida de uma pessoa (OLD, 2018).

Ainda segundo o autor Old (2018), os benefícios do Onco Coaching para a família e para o paciente vão desde assistir a passar pelo baque do diagnostico, trazer de volta a autoestima, apresentar resultados para minimizar os efeitos colaterais até maneiras de passar pelos tratamentos e fortalecer aptidões para enfrentar o problema.

Nestes momentos é onde o psicológico fica muito abalado, e a metodologia é apropriada para gerar uma maior aceitação de si mesma e o controle das emoções, trabalhando no desenvolvimento das habilidades e atitudes que as auxiliem a enfrentar o problema de uma maneira eficaz (MARQUES, 2014).

Para que um profissional possa ajudar um paciente com câncer, não precisa necessariamente ter formação na área da saúde, afirma o autor (ALVES, 2018).

De acordo com Longo (2015) a maioria dos médicos se sentem frustrados diante da incapacidade de curar o paciente e sofrendo com a pressão do horário totalmente preenchido deixam de prestar uma atenção mais cuidadosa e submetendo os pacientes somente a tratamentos paliativos. Ainda segundo o autor, além de medicamentos, gestos importantes acompanhados de cuidados e carisma como segurar a mão do paciente ou conversar com ele gera um conforto calmante. Na medicina, acredita-se que um bom estado mental influencia na produção de hormônios e na função do sistema imunológico e que ajuda muito o corpo a combater novos tumores.

O coaching mostra ao paciente o momento em que ele está vivenciando e escolher o que ele quer trabalhar. Não traça rotinas, ou faz uma lista de coisas sem antes saber se realmente é o que a pessoa quer (ALVES, 2018).

De acordo com os conteúdos analisados, verificou-se que o coaching tem um grande poder de atuar na inteligência emocional do paciente e lidar com suas emoções. O câncer além 
de problemas físicos, traz a desmotivação de muitos pacientes e profissionais. A maioria dos profissionais não sabem lidar com a efetividade ao tratar pacientes oncológicos deixando a desejar em muitos aspectos humanísticos de apoio e cuidados paliativos.

Outro aspecto analisado foi que pacientes com doenças oncológicas são leigos ao que diz respeito aos direitos a eles concedidos, podendo o coach auxiliar igualmente nessa área de acordo com Sonobe, Buetto \& Zago (2011).

A falta de comunicação também foi um fator relatado pelos pacientes por parte dos profissionais e que muitas vezes dificultam o relacionamento interpessoal contribuindo para a desmotivação no tratamento da doença.

Um coach que não é comunicativo raramente obtêm sucesso em seus encontros com o coachee/paciente. Lidar com o paciente oncológico e com suas emoções, é como ter em mãos uma pedra preciosa a ser lapidada onde requer todo cuidado, carinho e atenção.

\section{CONSIDERAÇÕES FINAIS}

O coaching apesar de não ser um estudo recente, atualmente vem se introduzindo de uma maneira mais abrangente em infinitas áreas de atuação.

Após a pesquisa, considera-se que o coaching além de diversas áreas em que atua, pode atuar como auxílio às pessoas portadoras de doenças oncológicas.

Os principais motivos que levam os clientes e organizações a procurarem o apoio de um coach estão no fato em que muitos se perderam em algum momento de uma etapa de suas vidas e precisam se reencontrar, seja na área profissional ou pessoal.

Ao mesmo tempo em que as áreas nas organizações estão adotando a metodologia como um diferencial no desenvolvimento de seus profissionais, outras áreas assim como a saúde, estão buscando se diferenciar adotando um novo papel e modelo de atenção aos pacientes.

À medida que crescem as demandas de tratamento, crescem juntamente as necessidades dos pacientes de atenção, apoio, segurança e estabilidade e percebe-se que muitos profissionais se sentem sobrecarregados de múltiplas tarefas rotineiras e não conseguem suprir essas necessidades. As estatísticas mencionam o câncer como a doença que mais mata pessoas no mundo todo e de certa forma, acabam acarretando um temor em quem sofre com esse dano.

O coaching deve ser apontado como uma solução, mostrando os impactos que pode causar na vida das pessoas antes, durante e após a sua prática ganhando a confiança do cliente. 
Como abordado na pesquisa, o coach tem como característica principal ser comunicativo, alto-astral, determinado, e corajoso ao assumir desafios. Viu-se também a importância de construir planos de ação para que se consiga chegar aos resultados almejados.

Na questão de avaliar o coaching como aliado ao tratamento de câncer foi relatado pelos autores citados nesse artigo, o árduo processo em que muitos pacientes passam juntamente com seus familiares desde o primeiro diagnósticos até as fases do tratamento e que muitas vezes se sentem mal informados, com pouco ou quase nenhum apoio efetivo por parte dos profissionais e que muitas vezes ficam desorientados sobre como procederem. Alguns autores relataram a laicidade de muitos pacientes em relação aos seus direitos legais, os quais advindos de informações de terceiros e não pelos profissionais da saúde. Outros pacientes sentem-se tão desorientados que não sabem o que mudar em suas rotinas diárias concernente a alimentação e hábitos.

E neste momento entra o papel do coach: orientar e traçar metas juntamente com o paciente para que o mesmo possa realizar um tratamento com mais qualidade de vida, foco na cura como resultado, e determinação no cumprimento das metas. O coach deve acompanhar todo o processo juntamente com seu coachee. $\mathrm{O}$ acesso as informações concernentes as leis que ampara o paciente oncológico, está ao alcance de todos e nada impede um profissional coach orientar ao seu cliente a respeito dos seus direitos.

E a confiança é um dos motivos que levam os clientes/pacientes a terem um relacionamento duradouro com seu coach. Quanto mais se sentirem motivados e seguros, com alguém que realmente consiga apontar seu potencial e mostrar que pode ir muito além, os pacientes evoluem em seu quadro clínico. Segundo o SBC Coaching as relações sociais geram um impacto imenso sobre a saúde até em momento conturbados como em tratamentos de câncer e de acordo com um estudo da Universidade do Texas, os relacionamentos são fonte de contentamento, compadecimento e desinteresse desde que haja afeição entre as partes.

Como limitação desta pesquisa, foram encontradas poucas referências sobre o assunto abordado especificamente, o que não impediu de confirmarem-se então os objetivos específicos deste estudo.

O objetivo geral do trabalho foi atingido, confirmando que o processo de coaching pode influenciar e impactar no tratamento de pessoas com câncer.

Enfim, como sugestão para estudos futuros, sugere-se novas pesquisas na área do coaching ampliando o conhecimento sobre o assunto incorporando igualmente na área da saúde mais especificamente nos casos realizados em institutos ou hospitais com pacientes oncológicos 
para descobrir como o coaching influencia no tratamento dos pacientes. Uma pesquisa prática também seria de grande valia.

\section{REFERÊNCIAS}

ALVES, Gabriel. Coaching oncológico ajuda paciente a lidar com medos e novas prioridades. Folha de São Paulo. Setembro de 2018. Disponível em: https://bit.ly/2w9kOis. Acesso em: 17 set. 2018.

BASSETTE, Fernanda. Cresce a mortalidade por câncer no país. Caso nenhuma medida seja tomada, calcula-se que até 2030 as neoplasias serão a primeira causa de óbito entre os brasileiros. Revista Veja. Abril de 2018. Disponível em: https://bit.ly/2Tpfbo2. Acesso em: 12 set. 2018.

BBC, Notícias. Dez gráficos que explicam o impacto do câncer no mundo. Fevereiro de 2016. Disponível em: https://bbc.in/383aupw. Acesso em: 12 set. 2018.

BOOG, Gustavo G.; BOOG, Magdalena. Manual de Treinamento e Desenvolvimento: processos e operações. $6^{\circ}$ ed. São Paulo: Pearson Education do Brasil, 2013. Disponível em: https://bit.ly/390ru0N. Acesso em: 11 set. 2018.

BRASIL. Ministério da Saúde. Secretaria de Atenção à Saúde. Instituto Nacional do Câncer. Coordenação de Prevenção e Vigilância. A situação do Câncer no Brasil. Rio de Janeiro; INCA; 2006. Disponível em: https://bit.ly/2HY45Be. Acesso em: 12 set. 2018.

CAMPOS, Claudinei José Gomes. Método de Análise de Conteúdo: ferramenta para a análise de dados qualitativos no campo da saúde. Revista Brasileira de Enfermagem, Brasília (DF) 2004 set/out; 57(5):611-4.

COSTA, Eva Cristina Boeno da; CAREGNATTO, Margareth Inês Motter. As percepções das empresas do ramo metalmecânico de Caxias do Sul sobre o desenvolvimento de seus profissionais por meio do coaching. Serra Gaúcha, 2012.

D’ADDARIO, Miguel. Coaching Pessoal. $3^{\circ}$ ed. CE. 2016. Disponível em: https://bit.ly/2VqVaQP. Acesso em: 12 set. 2018.

FRANÇA, Sulivan. O que é coching pessoal e como ele funciona? Entenda o que é o coaching pessoal, como ele funciona e quais são seus benéficos. Setembro de 2017.

Disponível em: https://bit.ly/2uzHsQu. Acesso em: 12 set. 2018.

GASPAR, Denis Juliano; PORTÁSIO, Renato Mehler. Liderança e Coaching:

Desenvolvimento pessoas, recriando organizações. Revista de Ciência gerenciais, vol. XIII, $\mathrm{n}^{\circ} 18,2009$.

G1. Portal G1. Coaching: O mercado que movimenta mais de U\$ 2,3 bilhões ao ano. G1. Coaching \& Desenvolvimento. 25 jun. 2018. Disponível em: https://glo.bo/395UqVe. Acesso em: 12 set. 2018.

GIL, Antônio Carlos. Métodos e técnicas de pesquisa social. 6. ed. - São Paulo: Atlas, 2008. 
JB. Jornal do Brasil. Câncer no Brasil pode aumentar em $78 \%$ nos próximos 20 anos. Jornal do Brasil. Ciência e Tecnologia. 12 set. 2018. Disponível em: https://bit.ly/2T4UDm4. Acesso em: 12 set. 2018.

JOSEFA, Marísia Silva. O coaching e o seu papel na melhoria do desempenho dos colaboradores nas organizações: Estudo exploratório na Empresa. Mindelo, 2015.

LAKATOS, Eva Maria; MARCONI, Marina de Andrade. Fundamentos de metodologia científica. 5. ed. São Paulo: Atlas, 2003. Disponível em: https://bit.ly/2Pw7RWD. Acesso em: 17 set. 2018.

LONGO, Dan L. Hematologia e Oncologia de Harrison. 18. ed. São Paulo: AMGH Editora LTDA, 2015. Disponível em: https://bit.ly/2Ty3lbB. Acesso em: 17 set. 2018.

LOTZ, Erika Gisele; GRAMMS, Lorena. Coaching e Mentoring. Curitiba: InterSaberes, 2014. Disponível em: https://bit.ly/2I3hgB4. Acesso em: 11 set. 2018.

LUBK, Rogério. Coaching: gerando transformações. São Paulo - INC Editora, 2016.

Disponível em: https://bit.ly/2I2VA7T. Acesso em: 11 set. 2018.

MALZYNER, Artur; CAPONERO, Ricardo. Câncer e Prevenção. $1^{\circ}$ ed. São Paulo: MG Editores. 2013. Disponível em: https://bit.ly/2vjZJS8. Acesso em: 12 set. 2018.

MARQUES, José Roberto. Quais os tipos de nichos de coaching? Janeiro de 2012. Disponível em: https://bit.ly/388PIo9. Acesso em: 24 jul. 2018.

MARQUES, José Roberto. História e tendências do coaching. Setembro de 2013. Disponível em: https://bit.ly/2T46wZr. Acesso em: 11 set. 2018.

MARQUES, José Roberto. Outubro Rosa: o coaching como apoio. Outubro de 2014. Disponível em: https://bit.ly/2T56kJt. Acesso em: 17 set. 2018.

MARQUES, José Roberto. Qual a origem do coaching? Abril de 2015. Disponível em: https://bit.ly/2vlGqrM. Acesso em: 11 set. 2018.

MARQUES, José Roberto. Coaching de Saúde - Nichos e Oportunidades.11 dez. 2015. Disponível em: https://bit.ly/2HZpZ7b. Acesso em: 12 set. 2018.

MARQUES, José Roberto. A Influência do Coaching nas Mudanças Comportamentais. Setembro de 2016. Disponível em: https://bit.ly/2w7qTMe. Acesso em: 12 set. 2018.

MARQUES, José Roberto. Conheça ferramentas de coaching efetivas para gerenciar crises. Maio de 2016. Disponível em: https://bit.ly/3c7U9Db. Acesso em: 11 set. 2018.

MARQUES, José Roberto. O que é Coaching pessoal e qual a diferença do Coaching profissional? Jun de 2016. Disponível em: https://bit.ly/3a7tNiL. Acesso em: 17 set. 2018.

MATTA, Villela da. Ferramentas de Coaching: Como Utilizá-las a Seu Favor? Junho de 2013. Disponível em: https://bit.ly/2PsXt23. Acesso em: 11 set. 2018. 
MATOS, Taína Camargo de. Coaching para profissional de saúde. Entenda a importância do processo de coaching na saúde pública do Brasil. 26 nov. 2017. Disponível em: https://bit.ly/2w92WUL. Acesso em: 12 set. 2018.

MUNHOZ, Antônio Siemsen. O coaching educacional no ensino a distância. Curitiba: InterSaberes, 2017. Disponível em: https://bit.ly/32vu24F. Acesso em: 11 set. 2018.

OLD, Dino. Onco Coaching chega no mercado para ajudar muitas pessoas. Revista EXAME. Maio de 2018. Disponível em: https://bit.ly/3acFuVc. Acesso em: 17 set. 2018.

ONEDA, Edmar. O que é coaching pessoal: O coaching pessoal, também chamado de Self Coaching, é um dos grandes nichos do Coaching. Março de 2016. Disponível em: https://bit.ly/2T5dvBg. Acesso em: 12 set. 2018.

PRODANOV, Cleber Cristiano; FREITAS, Ernani César de. Metodologia do Trabalho Científico: Métodos e Técnicas da Pesquisa e do Trabalho Acadêmico- 2. ed. - Novo Hamburgo: Feevale, 2013. Disponível em: https://bit.ly/2wSrqlD. Acesso em: 17 set. 2018.

SILVA, A.J.C.F. Coaching Psicológico: um estudo de casos. 55 p. Dissertação (Mestrado Integrado em Psicologia) - Faculdade de Psicologia, Universidade de Lisboa, 2012. Disponível em: https://bit.ly/383jb33. Acesso em: 17 set. 2018.

SOBRINHO, Wanderley Preite. Falta de médicos e de remédios: 10 grandes problemas da saúde brasileira. UOL Notícias. São Paulo. 09 mai. 2018. Disponível em:

https://bit.ly/2Tjr3bk. Acesso em: 12 set. 2018.

SONOBE, Helena Megumi; BUETTO, Luciana Scatralhe; ZAGO, Márcia Maria Fontão. $O$ conhecimento dos pacientes com câncer sobre seus direitos legais. Revista Escola de Enfermagem USP, São Paulo (SP) 2011, 45(2):342-8. Disponível em: https://bit.ly/32zbCjf. Acesso em: 18 set. 2018.

SPENCHLEY, Val; ROSENFIELD, Maxine. Tudo Sobre Câncer. São Paulo: Andrei. 2000. Disponível em: https://bit.ly/2PKd3GL. Acesso em: 12 set. 2018. 